\title{
LOS ALMENDROS (LA RODA): UN NUEVO YACIMIENTO PALEOLÍTICO EN ALBACETE
}

\author{
ALBERTO MINGO ÁLVAREZ, JESÚS BARBA REY Y MIGUEL ÁNGEL GARCÍA VALERO
}

Resumen. El yacimiento de Los Almendros se ubica en la localidad manchega de La Roda (Albacete). El material lítico estudiado fue recogido utilizando un sistema radial de sectorización que cubría 2500 metros cuadrados de una superficie problemática, con zonas de abundante monte bajo, y otras, más despejadas. En total se hallaron 1004 restos líticos, fundamentalmente en cuarcita. De ellos, 163 son útiles, destacando las raederas, lascas retocadas, y raspadores. Una primera aproximación a las características de la industria lítica nos ha permitido sugerir su atribución crono-cultural al tecno-complejo Musteriense.

Palabras clave. Los Almendros, Paleolítico, sistema radial de sectorización, industria lítica, Musteriense.

\begin{abstract}
The site of Los Almendros is located in La Roda (Albacete). The lithic material studied was gathered through a radial sectorization collecting system which covered a problematic surface of $2500 \mathrm{~m}^{2}$, including zones of abundant scrubland and others clearer. A total amount of 1004 lithic pieces were registred, mainly in quartzite, 163 of which were tools. The side-scrapers, retouched flakes, and scrapers are the most numerous. A first approach to the features of the lithic industry has allowed us to suggest the crono-cultural attribution of this site to a Mousterian period. Key words. Los Almendros, palaeolithic, radial collecting system, lithic industry, Mousterian.
\end{abstract}

\section{INTRODUCCIÓN}

La prospección arqueológica del término municipal de La Roda (Albacete) ha permitido localizar varios yacimientos atribuidos al Paleolítico Inferior y Medio (Fig. 1). El sitio de Los Almendros se encuentra al Sudoeste de este municipio, en una zona que presenta una topografía poco definida con grandes superficies de morfología llana, a una altitud de 750 metros. El terreno alberga una

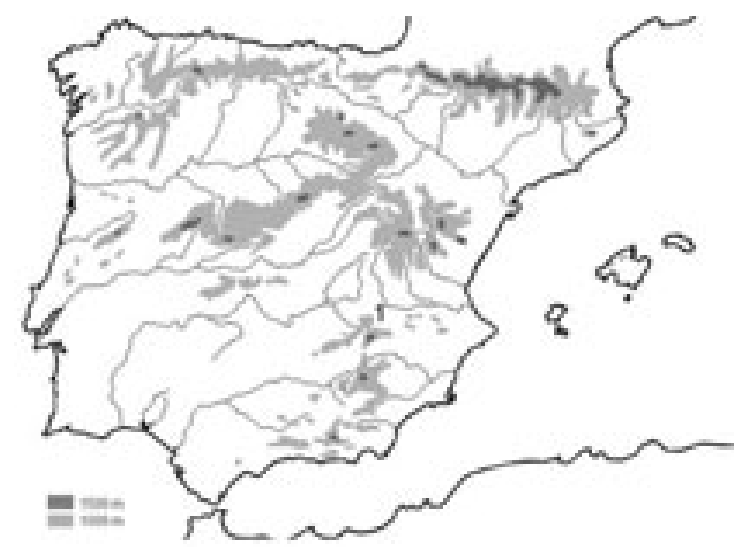

Figura 1: Situación del yacimiento de Los Almendros. vegetación compuesta principalmente por romero, aliagas y encinas que en ocasiones presentan porte arbóreo.

Desde el punto de vista geológico, el yacimiento se localiza en glacis con costra carbonatada laminar bandeada del Plioceno Superior (IGME 1988, 18) que constituyen el enlace entre el borde septentrional de la Plataforma Morfoestructural de Campo de Montiel y la Llanura Manchega. Esta formación es el último depósito generado durante el Villafranquiense en la región, estableciendo el límite entre éste y el Cuaternario.

La abundancia de restos líticos, así como la elevada concentración de los mismos, nos sugirió la posibilidad de plantear un sistema de recogida de material que permitiese establecer el área nuclear del yacimiento.

\section{TRABAJOS REALIZADOS}

El enclave de Los Almendros se encuentra en un suelo de escaso desarrollo, en el que en 


\begin{tabular}{|c|c|c|c|}
\hline ÁREAS & $\begin{array}{c}\text { M2 } \\
\text { (cada sector de cada anillo) }\end{array}$ & $\begin{array}{c}\text { \% de superficie total } \\
\text { (cada sector de cada anillo) }\end{array}$ & $\begin{array}{c}\text { \% superficie total anillos } \\
\text { (16 sectores de cada anillo) }\end{array}$ \\
\hline A & 5,27 & 0,25 & 4,05 \\
\hline B & 15,81 & 0,76 & 12,16 \\
\hline C & 26,45 & 1,27 & 20,34 \\
\hline D & 36,89 & 1,77 & 28,37 \\
\hline E & 45,58 & 2,19 & 35,06 \\
\hline TOTAL & 130 & 6,24 & 99,99 \\
\hline
\end{tabular}

Figura 2: Superficies de la sucesión de anillos y sectores.

ocasiones afloran costras carbonatadas, con una escasa intervención antrópica debido a su nulo aprovechamiento agrícola. La visibilidad de la superficie del terreno durante los trabajos de prospección fue buena, aunque la presencia de la vegetación calcícola dificultó puntualmente la visión en algunas zonas.

\subsection{Método}

El área que ocupaba la dispersión del material era aproximadamente de unos 2500 metros cuadrados. A tal efecto, se instaló un nivel topográfico en el centro de esta superficie (Fig. 3), con la finalidad de definir un punto cero del que partirían 16 ejes radiales equidistantes cada 25 grados, que a su vez se dividirían en cinco tramos de igual longitud, lo cual generaría un círculo central y cuatro anillos concéntricos al primero, subdivididos a su vez en sectores de morfología trapezoidal. Los anillos se denominaron de dentro a fuera $A, B, C, D$ y $E$, y los sectores se numeraron del 1 al 16 siguiendo la dirección de las agujas del reloj. De este modo, se generó un modelo radial que tomó forma mediante la colocación de cuerdas de color blanco para proporcionar una clara visualización entre la vegetación. Así, se definió un área de 2.080 metros cuadrados dividida en 80 sectores que presentaban 5 tipos de extensión dependiendo del anillo en el que se encuentre cada uno de aquellos (Fig. 2).

A continuación se procedió realizar los trabajos de prospección intensiva de cada sector por un equipo de cuatro arqueólogos, recogiendo todos los elementos líticos en bolsas debidamente identificadas.

\subsection{Análisis espacial}

En los sectores en los que existe abundante matorral o carrascas se localizó un menor número de elementos líticos, que probablemente encuentra su explicación en la dificultad de visibilidad que propiciaba la hojarasca del suelo. Un nuevo factor a incluir en esta biogénesis es la actividad de los lagomorfos, ya que en la realización de sus madrigueras contribuyen a la remoción del suelo. La mayor presencia de madrigueras coincide con los lugares donde hay más vegetación, en este caso carrascas y matorral bajo. El sector D4 es un exponente de toda esta alteración biogenética sobre el suelo.

Se localizó un antiguo camino, ya cubierto en parte de vegetación, que atraviesa longitudinalmente el área de estudio (atraviesa principalmente los sectores 9, 16, y la mitad oeste del anillo; y parcialmente los sectores 10 y 16). El camino tiene entre 3,5 y 5 metros de anchura y en esta zona aparecen muy pocos restos líticos, sin embargo en los márgenes se registra un mayor número debido con toda probabilidad a que en su día se retiraron las tierras para la confección del camino, aportando éstas en los laterales y por lo tanto procediéndose a una mayor acumulación de elementos líticos. El camino conforma una pequeña depresión aprovechada por una escorrentía poco acusada, que genera escasos cambios de cota, si bien ha contribuido a dar al camino una cierta morfología en cubeta. Esta circunstancia también la encontramos en los sectores E-3, E-4, E-5 y E-6, donde se encuentra una pista de uso actual, si bien no muy transitada, que afecta a estos sectores por su margen derecha. Esta afección en el caso de los sectores E-4 y E-5 es de un $80 \%$ de su superficie, mientras que en los sectores E-3 y E-6 era del 30\%.

En los sectores E-11 y E-12 el afloramiento de la costra caliza es notorio y se evidencia en mayor grado en áreas próximas al exterior de la zona de estudio, este hecho contribuyó sobremanera a que la visibilidad de la superficie del terreno fuese óptima, lo cual supuso que se recuperasen un mayor número de restos líticos.

No se documentan un importante número de piezas en todo el cuadrante 3, las áreas donde ligeramente se aprecian pequeñas concentraciones son el sector E-11, E-12, y algo en D-11 (Fig. 3). En todo el cuadrante hay, en general, 
poca vegetación ( 4 o 5 carrascas, tamaño medio pero sí algo de matorral bajo).

In situ, nos percatamos claramente de la existencia de una alta densidad de material lítico en el cuadro E-3. Parte de este cuadro, como ya indicamos anteriormente, coincide con una zona que ha sido remozada para adecuar la pista que atraviesa el lado oeste del área prospectada; la misma no alberga prácticamente ningún elemento sin embargo la zona no alterada es la que presenta la gran concentración.

Tanto sobre el terreno como en el croquis de densidad (Fig. 3) se observa en el primer cuadrante (sectores 1, 2, 3 y 4) un corredor de alta densidad de material lítico que transcurriría por los sectores C-3, D-3 y E-3 en su límite con los sectores número 2 , y ocuparía parte del sector $\mathrm{C}-4$. Coincide con un área desprovista de vegetación. Las zonas de carrascas próximas apenas ofrecen restos y se presentan cierta elevación como consecuencia de la materia orgánica.

En el cuadrante 2 (sectores 5-6-7-8) hay algunas concentraciones de alta densidad de material: en la zona libre de vegetación del C-7, en límite del sector E-7 con el E-8, y en el límite entre D-8 y D-9 (Fig. 3).

No se documenta un importante número de piezas en todo el cuadrante 3 (sectores 9, 10, 11 y 12) donde en general hay poca vegetación. Las áreas donde se aprecian pequeñas concentraciones y una mayor densidad de material son los sectores E-11, E-12, E-13 y D-12 (Fig. 3).

Por su parte, el cuadrante 4 (sectores 13, 14,15 y 16) fue el más pobre con una densidad muy baja de elementos líticos por metro cuadrado y $\sin$ apenas concentraciones significativas (Fig. 3).

\section{ANÁLISIS DE LOS MATERIALES}

El conjunto de restos líticos recuperados es bastante numeroso ascendiendo a los 1.004, de los cuales 709 son piezas tipológicamente adscribibles, mientras que los 296 restantes corresponden a debris (Fig. 4). En cuanto a las materias primas utilizadas para la confección de las piezas, destaca sobremanera la cuarcita de grano fino con un $92,38 \%$ (655 piezas), seguido por el sílex que supone el $4,23 \%$ (30 piezas), la caliza con un 2,54\% (18 piezas); por último contamos con cinco piezas de arenisca y una de cuarzo que representan el $0,85 \%$. De las 709 piezas presentan algún grado de corteza 314 (44,28\%), y por lo general tienen un escaso o nulo grado de rodamiento, con aristas vivas, sin embargo en

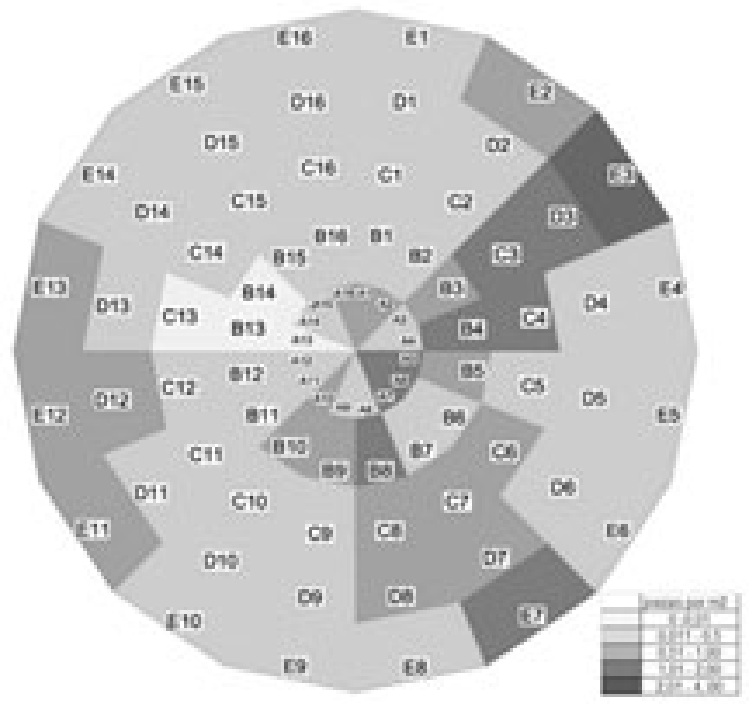

Figura 3: Densidad de los materiales sobre el modelo radial aplicado.

ocasiones incorporan concreciones de carbonatos como resultado del contacto con suelos muy calizos.

Las piezas no retocadas ascienden a 546 (Fig. 5), destacando las lascas con un 68,93\%. La alta constatación de núcleos (49), flancos de núcleo (29), fragmentos de canto (32), restos de talla o debris (296); y el relativamente bajo número de piezas retocadas (163), nos induce a considerar la posibilidad de que este enclave arqueológico se corresponda con un taller para la fabricación de útiles líticos.

La técnica levallois se encuentra escasamente representada con cinco lascas, una lasca seudo levallois, un núcleo, y alguna raedera (Fig. 8). Los núcleos suelen ser de extracción centrípeta y morfología globular, presentando bastante grado de aprovechamiento. Destaca sobremanera la total ausencia de útiles nucleiformes en un conjunto tan numeroso.
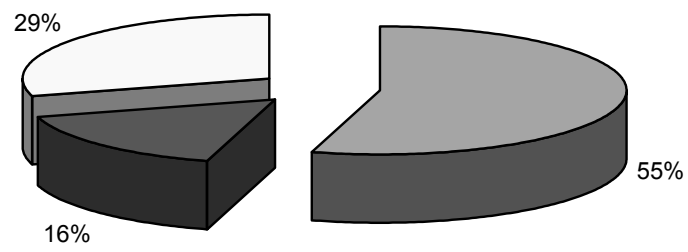

口NO RETOCADO $\square$ RETOCADO $\square$ DEBRIS

Figura 4: Categorías y representatividad del material. 


\begin{tabular}{|l|r|r|}
\hline \multicolumn{1}{|c|}{ ELEMENTO } & \multicolumn{1}{c|}{$\%$} & N \\
\hline CANTO & $0,37 \%$ & 2 \\
\hline CHUNK & $5,51 \%$ & 30 \\
\hline CUCHILLO DE DORSO & $2,21 \%$ & 12 \\
\hline FLANCO DE NÚCLEO & $5,33 \%$ & 29 \\
\hline FRAGMENTO DE CANTO & $5,88 \%$ & 32 \\
\hline LASCA LEVALLOIS & $0,92 \%$ & 5 \\
\hline LASCA PSEUDO-LEVALLOIS & $0,37 \%$ & 1 \\
\hline LASCAS & $68,93 \%$ & 375 \\
\hline NÚCLEOS & $9,01 \%$ & 49 \\
\hline PERCUTORES & $1,47 \%$ & 8 \\
\hline SEMITABLETAS & $0,37 \%$ & 1 \\
\hline TOTAL GENERAL & $\mathbf{1 0 0} \%$ & $\mathbf{5 4 6}$ \\
\hline
\end{tabular}

Figura 5: Representatividad del material no retocado.

Por lo que se refiere a los útiles retocados (Fig. 6) las raederas es el grupo más abundante con 75 piezas, lo cual supone un $46 \%$ del material retocado. Entre las raederas se encuentran algunos ejemplares tipo quina (Fig. 7). Los raspadores alcanzan un $14,11 \%$ con 23 ejemplares, lo que supone que tras las raederas y las lascas retocadas sea el grupo de útiles más abundante, seguido de lejos por el grupo de los denticulados (muescas y denticulados) que asciende a un $7,97 \%$.

La ausencia absoluta de útiles nucleiformes, el escaso tamaño de los útiles, junto con la presencia en un $60,12 \%$ de raederas y raspadores, son los aspectos que nos permiten atribuir este yacimiento a algún momento del Paleolítico medio (a falta de un estudio más detallado y exhaustivo del material que facilite su adscripción a una fase más concreta dentro de este tecnocomplejo), circunstancias a las que debemos sumar la presencia anecdótica de una punta musteriense (Fig. 9) y una punta levallois retocada.

Como se expuso más arriba, las características del conjunto lítico del yacimiento

\begin{tabular}{|l|r|r|}
\hline \multicolumn{1}{|c|}{ ELEMENTOS } & \multicolumn{1}{c|}{$\%$} & N \\
\hline LASCAS RETOCADAS & 30,06 & 49 \\
\hline DENTICULADOS & 5,52 & 9 \\
\hline MUESCAS & 2,45 & 4 \\
\hline PERFORADORES & 0,61 & 1 \\
\hline PUNTA SLEVALLOIS RETOCADAS & 0,61 & 1 \\
\hline PUNTAS MUSTERIENSES & 0,61 & 1 \\
\hline RAEDERAS & 46,01 & $\mathbf{7 5}$ \\
\hline RASPADORES & 14,11 & 23 \\
\hline TOTAL GENERAL & $\mathbf{1 0 0 \%}$ & $\mathbf{1 6 3}$ \\
\hline
\end{tabular}

Figura 6: Representatividad de los útiles.
Los Almendros contribuyen a considerar este enclave como un taller para la fabricación de útiles. La cuarcita con un $92,38 \%$ constituye la materia prima más utilizada, siendo el lugar de aprovisionamiento más cercano (escasos dos kilómetros) las terrazas del Pleistoceno Superior de la cañada de El Verdugal al norte de Los Almendros, que ofrecen gravas cuarcíticas y calizas procedentes de los afloramientos mesozoicos del Campo de Montiel. De igual modo, debemos tener en cuenta que el glacis en el que se localiza el yacimiento presenta en ocasiones cantos redondeados de cuarcita interestratificados entre encostramientos carbonatados (IGME 1988, 18), por lo que también ofrecen posibilidad de aprovechamiento como materia prima lítica.

En cuanto a la distribución de los materiales recuperados en la prospección del yacimiento destaca el sector E-3 con una densidad de 3,9 elementos por metro cuadrado y con concentraciones inusuales (Fig. 3) para yacimientos paleolíticos en superficie. La existencia de un antiguo camino que atraviesa la zona prospectada de norte a sur puede haber supuesto una modificación en el registro arqueológico, ya que se constata en su ámbito un descenso en las concentraciones con respecto a la zona adyacente oriental. Por su parte, la pista de uso actual que afecta por su margen derecha la zona estudiada, puede haber contribuido a la génesis de algunas acumulaciones exageradas de material lítico, como consecuencia del aporte de sedimento en los laterales del camino durante la realización del mismo, principalmente constatables en el sector E-3. Por todo lo anterior, podemos pronunciarnos respecto a que el área nuclear del yacimiento se encontraría en la zona-corredor definida por los sectores E-3, D-3, C-3, B-4 y C4 y al exterior del sector E-3. En esta zona nuclear no se aprecian diferencias porcentuales con el resto de los sectores en cuanto a representación de piezas y debris.

De igual modo, habría otras áreas con menor densidad que la citada ud supra, como son el extremo occidental (sectores E-11, E-12, E-13 y D-12) y el sud-oriental (sectores B-8, B9, B-10, C-6, C-7, C-8, D-7, D-8, E-7) que podrían corresponderse con áreas secundarias 0 menos utilizadas en mayor medida que a procesos postdepositacionales, especialmente si tenemos en cuenta la falta de una conexión homogénea entre estas zonas y el área nuclear observada. 


\section{EL CONJUNTO ARQUEOLÓGICO DE LOS ALMENDROS EN SU CONTEXTO REGIONAL}

Las labores de prospección del municipio de La Roda han puesto de manifiesto la existencia de otros enclaves con materiales atribuidos al $\mathrm{Pa}$ leolítico medio, sin embargo los más cercanos al que nos ocupa y en un radio de unos 500 metros, son, al noroeste, Las Tasoneras y al sur, Los Morteros y Cerro de la Cañada de Santa Marta. Estos sitios han aportado un menor número de restos líticos. Su estudio de conjunto con Los Almendros será objeto de otros trabajos.

El grueso de los yacimientos paleolíticos descubiertos hasta los años noventa en la provincia de Albacete se circunscriben a los ejes fluviales de tres zonas: la cuenca alta del Guadiana, la cuenca del río Mundo y las depresiones de Tus y el Zumeta (Sanz Gamo 1989:41). El caso de Los Almendros se relacionaría directamente con la cuenca alta del Guadiana, un área en la que las primeras referencias son los materiales localizados por A. Pérez González en el ámbito de los ríos Záncara, Rus, Córcoles y Alto Guadiana durante la elaboración de las hojas del mapa geológico de Villarrobledo y Minaya y posteriormente estudiados por M. Santonja (Santonja et alii, 1977; Santonja, 1981, a y b). Estos sitios arqueológicos fueron reestudiados a finales de los años noventa por J. L. Serna (1999), siendo los yacimientos denominados Pajarón y Malagana, situados a unos veinte kilómetros al sudoeste de Los Almendros, los más cercanos.

El Pajarón y Malagana han ofrecido exiguas colecciones de material (39 y 34 piezas respectivamente) realizadas en su totalidad sobre cuarcita. Se trata de materiales recogidos en la superficie de terrazas fluviales caracterizadas por ofrecer mantos de guijarros. En el Pajarón se documenta escasamente la técnica levallois y de la escasa muestra se puede señalar la alta proporción de piezas con muesca y denticulados (Serna, 1999, 122). Malagana presenta un alto porcentaje de núcleos con un $55,8 \%$, inexistencia de técnica levallois y presencia notable de cantos trabajados (Serna, 1999, 130). Ambos conjuntos, han sido, a pesar de la escasez de las piezas halladas, tipológicamente adscritos al Paleolítico medio.

Las reducidas colecciones de los citados yacimientos dificultan cualquier comparación con Los Almendros, si bien los núcleos y las lascas sin retocar suponen en los tres enclaves más del $60 \%$ del material recuperado, por lo que con toda probabilidad se trata de lugares destinados a la fabricación de útiles líticos. El estudio de los materiales recuperados en los cercanos emplazamientos de Las Tasoneras, Los Morteros y Cerro de la Cañada de Santa Marta junto con el enclave de Los Almendros ofrecerá, sin duda alguna, más información relevante sobre el poblamiento Paleolítico manchego, cuya escasez en el estado actual de nuestros conocimientos se debe más a la ausencia de líneas de investigación o prospecciones sistemáticas que a un desierto poblacional durante aquel período. En este sentido, creemos que el planteamiento de un proyecto interdisciplinar de estudio de la globalidad de estos yacimientos permitirá identificar e interpretar de una forma más precisa el poblamiento paleolítico de esta región.

\section{Dibujo de los materiales}
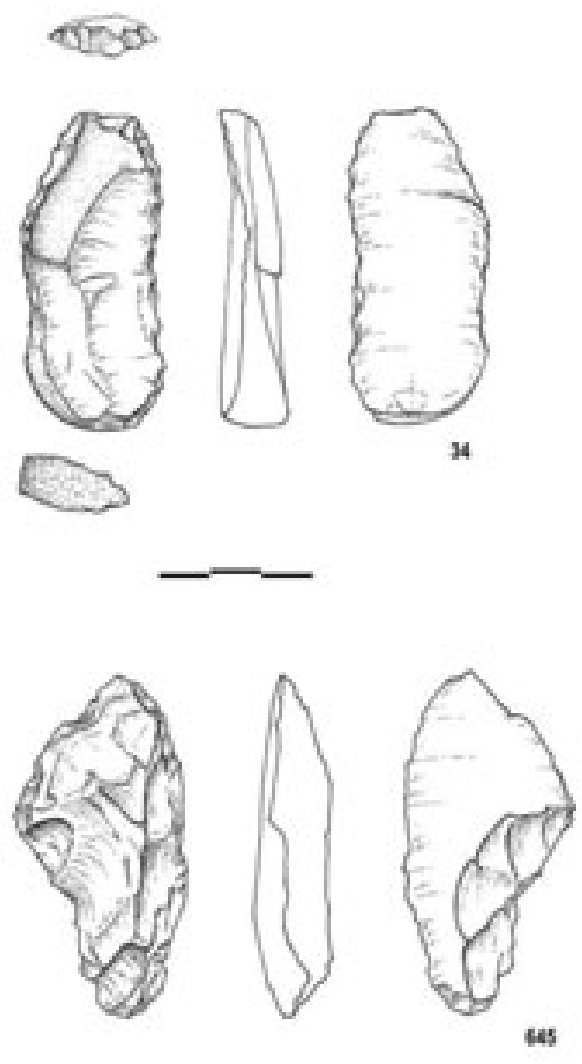

Figura 7: 34. Raspador. 645. Raedera. 

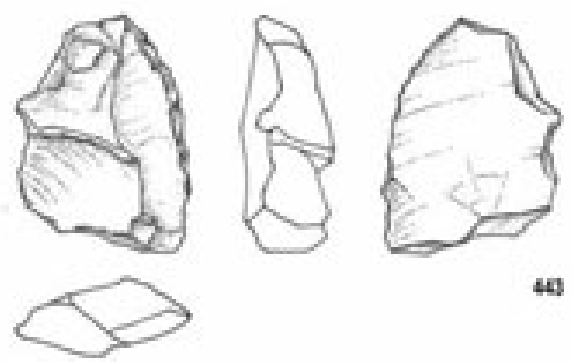

46
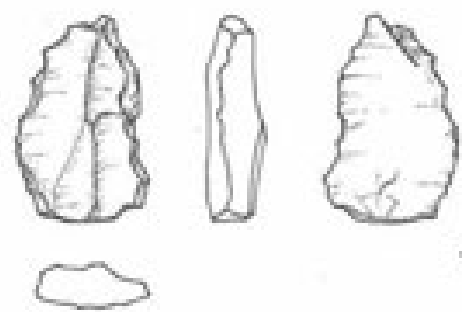

18

Figura 8: 443. Raedera-denticulado; 18. Raedera.

\section{Agradecimientos}

Queremos agradecer la colaboración para la realización de este trabajo a Noemí Morán, José Enrique Benito, Enrique Daza, Ricardo Barbas, José Antonio Galante, y Raquel Aliaga.

Prof. Alberto Mingo Álvarez Jesús Barba Rey

Dpto. de Prehistoria y Arqueología

UNED

c/ Senda del rey, 7

28040 Madrid

amingo@geo.uned.es

jbarba@bec.uned.es

Miguel Ángel García Valero

Gestión de Patrimonio de

la Delegación de Cultura de Guadalajara

PI. de San Esteban, 1

19071 Guadalajara

magarciav@jccm.es
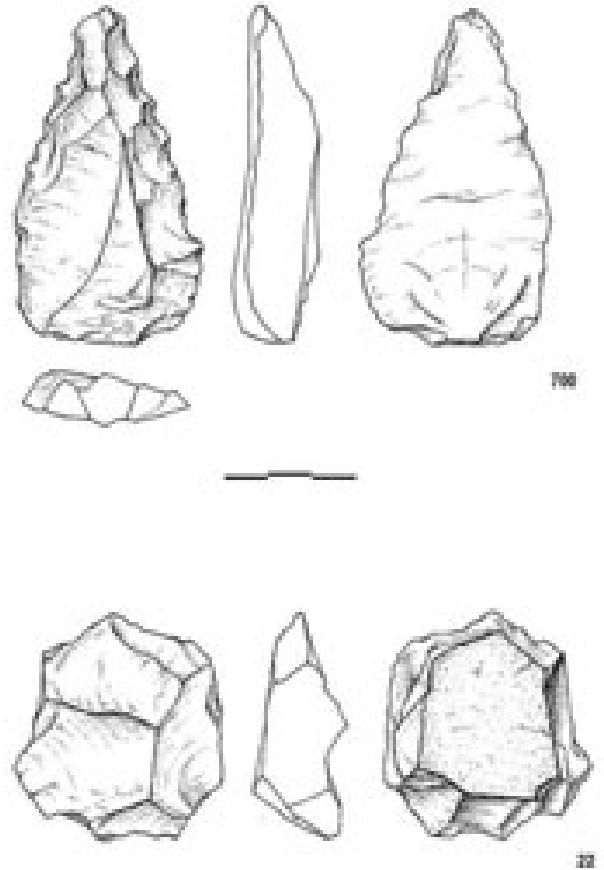

Figura 9: 700. Punta musteriense; 22. Núcleo discoide.

\section{BIBLIOGRAFÍA}

I.G.M.E., 1988: Mapa geológico de España, escala 1:50.000, hoja 764 (Munera). Madrid.

SANTONJA M. et alii., 1977: "Nuevas industrias paleolíticas en la Cuenca Alta del Guadiana: Estudio preliminar". Actas de la II Reunión del Grupo de Trabajo del Cuaternario. Jaca 1975.

SANTONJA, M., 1981 a: El Paleolítico Inferior en la Meseta Española. Tesis doctoral. Universidad Complutense.

SANTONJA, M., 1981 b: "Características generales del Paleolítico Inferior en la Meseta Española”. Numantia 1981.

SANZ GAMO, R., 1989: Museo de Albacete. Junta de Comunidades de Castilla-La Mancha.

SERNA LÓPEZ, J. L., 1999: El Paleolítico Medio en la provincia de Albacete. Instituto de Estudios Albacetenses "Don Juan Manuel". Excma. Diputación de Albacete. Serie I, Estudios, num. 91. 